\title{
Socioeconomic status and the association between height and obesity amongst first class primary school children in Ireland
}

\author{
A. Scully ${ }^{1}$, M.M. Heinen ${ }^{1}$, S. Bel-Serrat ${ }^{1}$, L. Daly ${ }^{2}$, J. Mehegan $^{2}$, \\ C.M. Murrin ${ }^{1}$ and C.C. Kelleher \\ ${ }^{1}$ National Nutrition Surveillance Centre, School of Public Health, Physiotherapy \& Population Science, \\ University College Dublin (UCD), Dublin, Ireland and ${ }^{2}$ School of Public Health, Physiotherapy \& Population Science, \\ UCD, Dublin, Ireland
}

Height can be used as a proxy for a child's growth and development and hence is strongly positively correlated with environmental factors including diet, income, education and health ${ }^{(1)}$. On the other hand, obese children tend to be taller before puberty compared to their normal weight peers ${ }^{(2)}$. In addition, family socioeconomic status (SES) generally shows a negative association with overweight and obesity in children ${ }^{(3)}$. So, it seems that an interactive effect exists between SES, height and childhood obesity, which we would like to explore more in the Irish part of the Childhood Obesity Surveillance Initiative (COSI).

This study used data collected in 2010, 2012 and 2015 as part of COSI. Primary school children from 1 st class $(\mathrm{n}=2691 ; 51.9 \%$ girls) partook in the study. Height and weight were measured in each child, following a standardised protocol ${ }^{(4)}$. Children's body mass index (BMI) was categorised using International Obesity Task Force (IOTF) cut-offs, and families completed a questionnaire to assess their family SES. ANOVA was performed for height and stratified by both BMI and SES categories. The results were presented for boys and girls separately. This resulted in low subject numbers for some of the BMI categories and categories with $\mathrm{n}<5$ were ignored.

Boys were on average $124.4 \mathrm{~cm}$ tall and girls $122.6 \mathrm{~cm}$. As expected, height differed significantly across the BMI categories $(\mathrm{p}<$ 0.05 ) for all SES categories, whereby obese children were consistently taller than underweight and normal weight children. Overall, there was no real indication of an interactive effect with SES, as height was similar between the different SES categories or differences were small. For example, for boys whose parents owned a house, height was $129.9 \mathrm{~cm}$ for obesity, $127.9 \mathrm{~cm}$ for overweight, $124.1 \mathrm{~cm}$ for normal and $122.1 \mathrm{~cm}$ for underweight. Whereas for boys whose parents rented a house, heights were only slightly lower: $128.8 \mathrm{~cm}$ for obese, $127.2 \mathrm{~cm}$ for overweight, $123.1 \mathrm{~cm}$ for normal and $121.6 \mathrm{~cm}$ for underweight.

Overall, the analysis confirms the positive association of height and overweight and obesity in both girls and boys in COSI, but there was no indication of an interactive effect with SES.

1. Gurzkowska B, Kulaga Z, Litwin M et al. (2014) Eur J Pediatr 173, 45-52.

2. Cecil JE, Watt P, Murrie ISL et al. (2005) Int J Obes 29, 1199-203.

3. Suchomlinov A \& Tutkuvience J (2016) J Biosoc Sci 48, 502-29.

4. Wijnhoven T \& Branca F (2008) WHO European Childhood Obesity Surveillance Initiate. Protocol, Version January 2008. Copenhagen: WHO Regional Office for Europe. 